\title{
Genetic information metabolism
}

Victor V. Tetz, George V. Tetz

\section{Source}

Victor V. Tetz, George V. Tetz. (2020). A new biological definition of life. doi:10.1515/bmc2020-0001.

the process responsible for, and involved in, DNA and RNA replication, methylation, repair, mutation, transcription, recombination, survival, and their spreading in both unicellular and multicellular organisms. 